\title{
The prognostic value and mechanisms of IncRNA UCAI in human cancer
}

This article was published in the following Dove Press journal:

Cancer Management and Research

\author{
Fei Yao \\ Qiang Wang' \\ Qingming $W u^{1,2}$ \\ 'Department of Public Health and \\ Preventive Medicine, School of Medicine, \\ Wuhan University of Science and \\ Technology, Wuhan 430065, People's \\ Republic of China; ${ }^{2}$ Hubei Province Key \\ Laboratory of Occupational Hazard \\ Identification and Control, Wuhan \\ University of Science and Technology, \\ Wuhan 430065, People's Republic of \\ China
}

\begin{abstract}
Long noncoding RNAs (lncRNAs), longer than 200 nucleotides in length, play important roles in the development and progression of various cancers. An increasing number of studies have revealed that lncRNAs function as potential oncogenes or tumor suppressors to influence biological processes, such as cell growth, invasion, migration and apoptosis. Urothelial carcinoma associated 1 (UCA1), an oncogenic lncRNA, was first found in bladder cancer and highly expressed in multiple cancers, including gastric cancer, colorectal cancer, lung cancer and breast cancer. UCA1 promotes tumorigenesis mainly via binding to tumor-suppressive microRNAs (miRNAs), activating several pivotal signaling pathways and alteration of epigenetic and transcriptional regulation. In addition, high expression of UCA1 is related to poor clinicopathological features especially for shorter overall survival, suggesting that UCA1 might be regarded as a prognosis biomarker in human cancers. In the present review, we summarized current studies on UCA1 to explore its prognostic value and underlying regulation mechanisms in the development of multiple cancers in order to provide a glimmer of hope for the prevention and treatment of malignant tumors.
\end{abstract}

Keywords: UCA1, lncRNA, cancer, prognostic, mechanism

\section{Introduction}

Cancer is one of the most common malignant diseases, with high morbidity and mortality, which seriously endanger people's health. ${ }^{1}$ The majority of patients are diagnosed in the advanced stage of the disease due to indiscernible early symptoms and lack of efficacious screening methods. ${ }^{2}$ In the present, there are various modes of treatment for cancer patients, including surgery, radiotherapy, chemotherapy, biotherapy and targeted therapy. ${ }^{3}$ However, the 5 -year survival rate remains dismal. ${ }^{4}$ Therefore, it is particularly important to identify new early biomarker and potential regulation mechanisms in the development of human cancers to improve the prognosis of patients. Recently, studies have shown that lncRNAs play important roles in tumorigenesis and prognosis via functioning as potential oncogenes or tumor suppressor genes. ${ }^{5-7}$

Less than $2 \%$ of the human genes can encode proteins, and the majority of the genome can be transcribed into non-coding RNAs, which further divided into long non-coding RNA (lncRNAs) and short non-coding RNAs (sncRNAs) according to their length. ${ }^{8,9}$ lncRNAs, lacking protein-coding capacity, are a class of non-coding RNA which are longer than 200 nucleotides in length and can modulate gene expression at the transcriptional, posttranscriptional, epigenetic and translation levels. ${ }^{10-12}$ Accumulating studies have found that lncRNAs are abnormal expressed
Correspondence: Qingming Wu School of Medicine, Wuhan University of Science and Technology, Huangjiahu West Road, Hongshan District, Wuhan City, Hubei 430065, People's Republic of China Tel +8618802705686

Email wuhe9224@sina.com 
and related to the prognosis in a variety of malignant tumors. Hence, it is necessary to explore whether lncRNAs can be used as prognostic markers and the mechanisms that affect the process of malignant tumors.

Urothelial carcinoma associated 1 (UCA1), a novel vital oncogenic lncRNA and a member of the human endogenous retrovirus $H$ family, is 1,442 bp in length and includes 3 exons and 2 introns. ${ }^{13}$ It was found that UCA1 is located on chromosome 19p13.12 with a TATA box at its $5^{\prime}$ end and a polyA tail at the $3^{\prime}$ end. ${ }^{14}$ According to the tissue expression profiling, UCA1 was ubiquitously expressed in embryonic tissues and not expressed in most normal tissues of adults (except the heart and spleen). ${ }^{15}$ UCA1 was firstly studied in bladder cancer and most localized in the cytoplasm, which may be crucial for the regulation of protein synthesis process in downstream genes. $^{16,17}$ In recent years, many reports focus on UCA1 and tumorigenesis, and have shown that UCA1 is highly expressed and exerts oncogenic activity in numerous cancers, such as gastric cancer, colorectal cancer, cervical cancer and melanoma. Several studies further indicated that highly expressed UCA1 might serve as a prognostic marker for cancer patients because it is correlated with advanced TNM stage, positive lymph node metastases, higher cell viability and migration, stronger chemotherapy resistance and shorter overall survival. ${ }^{18-20}$ In this review, we searched recent studies on UCA1 and summarized the underlying mechanisms and relevant clinicopathological features in human cancers, as shown in Tables 1 and 2, to explore the prognostic value of UCA1.

\section{UCAI in human cancers}

Accumulating studies have demonstrated that UCA1 is highly expressed in various cancers, and its upregulation promotes cellular functions, such as cell proliferation, migration and invasion, which are closely related to prognosis of patients. The present review generalized the prognostic value and underlying mechanisms of UCA1 in human cancers.

\section{Bladder cancer}

UCA1, first studied in bladder cancer, was found to be overexpressed in bladder cancer (BC) cell lines. Previous studies have shown that HMGB1 can act as an inducer of epithelial-mesenchymal transition (EMT) to influence the invasion ability of BC cells. ${ }^{21,22}$ Xue et al demonstrated overexpression of UCA1 could induce EMT process in two ways, the one is through increasing the levels of zinc finger E-box binding homeobox 1 and 2 (ZEB1/2), another way is by inhibiting tumor suppressor hsa-miR -145 and then increasing the level of fascin homolog 1 (FSCN1) to promote the migration and invasion of bladder cancer cells. ${ }^{23}$ Luo et al also reported that UCA1 inhibited tumor suppressor miR-143 and then upregulated the expression of HMGB1 to promote invasion of bladder cancer cells. ${ }^{24}$ In addition, Gou et al reported that BMP9 was highly expressed in superficial and infiltrating bladder cancer. Furthermore, the researchers found that increased BMP9 markedly upregulated the expression of UCA1, thereby facilitating the proliferation and migration of $\mathrm{BC}$ cell. $^{25}$ Therefore, these data suggested that UCA1 may enhance $\mathrm{BC}$ progression by regulating proliferation and migration ability of cancer cells.

\section{Colorectal cancer}

Several studies have found that when compared with noncancerous tissues and normal colorectal cells, UCA1 was upregulated in colorectal cancer (CRC) tissues and cell lines. According to previous reports, lncRNA could act as a competing endogenous RNA (ceRNA) to regulate RNA molecules in cytoplasmic. ${ }^{26-29}$ Bian et al suggested that amplified UCA1 promoted cell proliferation and tumorigenicity, the potential mechanisms were explored and found that UCA1 sponged miR-204-5p to enhance tumor-promoting genes CREB1, BCL2 and RAB22A expression. $^{30} \mathrm{Ni}$ et al analyzed the relationship between UCA1 and clinicopathological features, and found that expression of UCA1 in CRC was significantly associated with positive lymphatic metastasis and higher tumor stage. Kaplan-Meier analysis results indicated that patients with high UCA1 expression had a significantly poorer prognosis compared to those with low UCA1 expression. Then, the author further reported silencing UCA1 repressed cell proliferation, migration, invasion and promoted apoptosis. ${ }^{31}$ These studies strongly suggested that increased UCA1 exerted oncogenic effects in CRC.

\section{Gastric cancer}

The function and mechanisms of UCA1 were investigated in gastric cancer (GC). Li et al assessed the biological function of UCA1 by overexpression or downregulation of UCA1 in gastric cancer cell lines. Experimental results showed that highly expressed UCA1 enhanced the expression of p-AKT3, p-mTOR and S6K and promoted proliferation, migration and invasion of $\mathrm{GC}$ cells, while silencing of UCA1 have the opposite results, indicating 
Table I Functional characterizations of UCAI in multiple human cancers

\begin{tabular}{|c|c|c|c|c|c|}
\hline Cancer types & Expression & Role & Function role & Related genes & References \\
\hline Bladder cancer & Upregulated & Oncogene & $\begin{array}{l}\text { Cell proliferation, migration, } \\
\text { invasion }\end{array}$ & $\begin{array}{l}\text { miR-I43, HMGBI, miR-195, ZEBI/2, hsa-miR-I45, } \\
\text { FSCNI, BMP9, ARL2 }\end{array}$ & $23-25$ \\
\hline $\begin{array}{l}\text { Colorectal } \\
\text { cancer }\end{array}$ & Upregulated & Oncogene & $\begin{array}{l}\text { Cell proliferation, migration, } \\
\text { invasion, cell antiapoptosis }\end{array}$ & miR-204-5p,CREBI, BCL2, RAB22A & 30,31 \\
\hline Gastric cancer & Upregulated & Oncogene & $\begin{array}{l}\text { Cell proliferation, migration, } \\
\text { invasion, cell antiapoptosis }\end{array}$ & $\begin{array}{l}\text { miR-I82, TIMP2, Bcl-2, Bax, ZEB2, miR-203, miR- } \\
\text { 590-3p, CREBI, PI3K/Akt/mTOR, PI3K/AKT/ } \\
\text { GSK3ß, NF-KB signaling pathway }\end{array}$ & $32-35$ \\
\hline $\begin{array}{l}\text { Hepatocellular } \\
\text { cancer }\end{array}$ & Upregulated & Oncogene & $\begin{array}{l}\text { Cell proliferation, migration, } \\
\text { invasion, cell cycle control }\end{array}$ & $\begin{array}{l}\text { miR-2 I6b, FGFRI, snail, miR-203, EMT, ERK signal- } \\
\text { ing pathway }\end{array}$ & 38,39 \\
\hline Breast cancer & Upregulated & Oncogene & $\begin{array}{l}\text { Cell proliferation, migration, } \\
\text { metastasis, chemoresistance }\end{array}$ & $\begin{array}{l}\text { miR-I8a, YAPI, PTEN, mTOR, Wnt/ } \beta \text {-catenin sig- } \\
\text { naling pathway }\end{array}$ & $40-42$ \\
\hline $\begin{array}{l}\text { Esophageal } \\
\text { cancer }\end{array}$ & Upregulated & Oncogene & Cell proliferation, migration & miR-204, SOX4 & 43 \\
\hline Lung cancer & Upregulated & Oncogene & Cell proliferation, invasion & miR-I93a-3p, ERBB4, HMGBI & 45,46 \\
\hline $\begin{array}{l}\text { Prostate } \\
\text { cancer }\end{array}$ & Upregulated & Oncogene & $\begin{array}{l}\text { Cell cycle control, radio and } \\
\text { chemo-resistance }\end{array}$ & miR-204, sirtI, PI3K/AKT pathway & 47,48 \\
\hline $\begin{array}{l}\text { Pancreatic } \\
\text { cancer }\end{array}$ & Upregulated & Oncogene & $\begin{array}{l}\text { Cell proliferation, migration, } \\
\text { invasion, cell antiapoptosis }\end{array}$ & miR-I35a, miR-96, FOXO3, p27 & $49-51$ \\
\hline Ovarian cancer & Upregulated & Oncogene & Chemo-resistance & $\operatorname{miR}-129, A B C B I$ & 52,53 \\
\hline $\begin{array}{l}\text { Tongue } \\
\text { carcinoma }\end{array}$ & Upregulated & Oncogene & Cell migration, invasion & TGF- $\beta I$, miR-I24, JAGI, Notch I & 54,55 \\
\hline Melanoma & Upregulated & Oncogene & $\begin{array}{l}\text { Cell proliferation, metastasis, } \\
\text { invasive, cell cycle control }\end{array}$ & miR-507, FOXM & 56,57 \\
\hline $\begin{array}{l}\text { Thyroid } \\
\text { carcinoma }\end{array}$ & Upregulated & Oncogene & $\begin{array}{l}\text { Cell proliferation, invasion, } \\
\text { cell antiapoptosis }\end{array}$ & miR-204, IGFBP5, Wnt/ $\beta$-catenin pathway & 58,59 \\
\hline Osteosarcoma & Upregulated & Oncogene & $\begin{array}{l}\text { Cell proliferation, invasion, } \\
\text { cell cycle control }\end{array}$ & $\begin{array}{l}\text { HIF-I } \alpha, \text { miR-30Ia, CXCR4, PTEN/AKT, Wnt/ } \beta \text { - } \\
\text { catenin, NF-кB pathway }\end{array}$ & 60,61 \\
\hline Glioma & Upregulated & Oncogene & $\begin{array}{l}\text { Cell proliferation, migration, } \\
\text { invasion, cell antiapoptosis }\end{array}$ & miR-I22, miR-I82, iASPP & 62,63 \\
\hline $\begin{array}{l}\text { Multiple } \\
\text { myeloma }\end{array}$ & Upregulated & Oncogene & $\begin{array}{l}\text { Cell proliferation, cell } \\
\text { antiapoptosis }\end{array}$ & TGF- $\beta$ & 64 \\
\hline $\begin{array}{l}\text { Oral squamous } \\
\text { cell carcinoma }\end{array}$ & Upregulated & Oncogene & $\begin{array}{l}\text { Cell proliferation, migration, } \\
\text { invasion, cell antiapoptosis, } \\
\text { chemo-resistance }\end{array}$ & miR-I84, SFI, Wnt/B-catenin pathway & 65,66 \\
\hline $\begin{array}{l}\text { Cervical } \\
\text { cancer }\end{array}$ & Upregulated & Oncogene & $\begin{array}{l}\text { Cell proliferation, migration, } \\
\text { invasion, radio-resistance }\end{array}$ & miR-206, VEGF, HK2, glycolytic pathway & 67,68 \\
\hline
\end{tabular}

UCA1 affected cellular functions in GC partly through activation of PI3K-Akt-mTOR signaling pathway. ${ }^{32}$ Then, Qin et al similarly indicated UCA1 was overexpressed in gastric cancer tissues and cell lines. The level of miR-182 was downregulated and its target gene TIMP2 that closely related to cell proliferation and apoptosis was significantly increased when knockdown UCA1, which decreased the expression of Bcl-2 while enhanced the 
Table 2 Clinical significance of UCAI in various human cancers

\begin{tabular}{|c|c|c|}
\hline Cancer types & Clinicopathological features & References \\
\hline Colorectal cancer & $\begin{array}{l}\text { Positive lymphatic metastasis, advanced tumor stage, larger tumor size, greater depth of invasion, } \\
\text { poorer overall survival }\end{array}$ & 30,31 \\
\hline Gastric cancer & Advanced TNM stage, higher invasion depth, positive lymph node metastasis, shorter overall survival & 32,35 \\
\hline Hepatocellular cancer & Advanced TNM stage, larger tumor size, positive vascular invasion, shorter overall survival & 38,39 \\
\hline Esophageal cancer & Advanced TNM stage, higher differentiation, positive lymph node metastasis, shorter overall survival & 43 \\
\hline Lung cancer & Advanced TNM stage, larger tumor size, shorter overall survival & 45 \\
\hline Pancreatic cancer & Shorter overall survival & 51 \\
\hline Ovarian cancer & $\begin{array}{l}\text { Advanced TNM stage, positive lymph node metastasis, shorter overall survival, resistance to } \\
\text { chemotherapy }\end{array}$ & 52,53 \\
\hline Tongue carcinoma & $\begin{array}{l}\text { Advanced TNM stage, positive lymph node metastasis, greater depth of invasion, poorer overall } \\
\text { survival }\end{array}$ & 54,55 \\
\hline Melanoma & Positive lymph node metastasis & 56 \\
\hline Thyroid carcinoma & Advanced TNM stage, larger tumor size & 58 \\
\hline Glioma & Advanced TNM stage, larger tumor size, positive lymph node metastasis, shorter overall survival & 62,63 \\
\hline Multiple myeloma & Shorter overall survival & 64 \\
\hline $\begin{array}{l}\text { Oral squamous cell } \\
\text { carcinoma }\end{array}$ & Advanced TNM stage, positive lymph node metastasis & 65 \\
\hline
\end{tabular}

level of Bax. Further research revealed the expression of p-PI3K, p-AKT and p-GSK3 $\beta$, key factors of PI3K/AKT/ GSK3 $\beta$ signal pathways, and $\mathrm{p}-\mathrm{I \kappa B} \alpha$ and $\mathrm{p}-\mathrm{p} 65$, main factors of NF- $\mathrm{KB}$ signal pathway, was increased when overexpression miR-182. ${ }^{33}$ In addition, Gong et al indicated that upregulated UCA1 facilitated to cell migration and invasion in GC patients. Mechanistically, the researchers found that UCA1 contributed to GC progression via competitively binding miR-203 to increase the expression of ZEB2. ${ }^{34}$ Similarly, Gu et al also discovered that UCA1 sponged miR-590-3p to enhance CREB1 expression, which would promote GC progression in vitro and vivo. ${ }^{35}$ In short, these findings revealed that UCA1 might facilitate to the development of GC.

\section{Hepatocellular carcinoma}

Several meta-analyses have concluded that lncRNA UCA1 acted as a predictor of poor overall survival for patients with hepatocellular carcinoma (HCC). ${ }^{36,37}$ Wang et al showed that upregulation of UCA1 was markablely related with advanced TNM stages, positive metastasis and poorer survival rate. After further evaluation of biological function, the proliferation, migration and invasion capacity of
HCC cell was repressed, and cell cycle was arrest in G0/ G1 when knockdown UCA1. Subsequently, bioinformatics analysis and luciferase assays revealed that UCA1 could function as an endogenous sponge of miR-216b to promote FGFR1 expression. Depletion of UCA1 repressed the protein expression levels of ERK1/2 and p-ERK1/2, suggesting extracellular signal-regulated kinase (ERK) signaling pathway was involved in $\mathrm{HCC}$ progression. ${ }^{38}$ In addition, Xiao et al similarly demonstrated that silencing UCA1 inhibited HCC cell invasion and metastasis in vitro and in vivo. Moreover, UCA1 could competitively bind to miR-203 to enhance the expression of snail, promoting epithelial-mesenchymal transition and tumor progression. ${ }^{39}$ Consequently, these data indicated that UCA1 could serve as an oncogene in tumorigenesis.

\section{Breast cancer}

Several studies have identified the relationship between UCA1 and chemosensitivity of breast cancer (BC) cells. A report by Liu et al confirmed the strong association between UCA1 and tamoxifen resistance. Mechanistically, inhibition of UCA1 significantly attenuated the proliferation, migration and chemoresistance in the tamoxifen-resistant 
MCF-7-R and T47D-R cells, possibly due to inhibition of the $\mathrm{Wnt} / \beta$-catenin pathway activation. Because further research identified that knockdown UCA1 prevented the nuclear translocation of $\beta$-catenin. ${ }^{40}$ Similarly, Wu et al suggested enforced UCA1 markedly reduced tamoxifen sensitivity of MCF-7 cells through activating mTOR signaling pathway. ${ }^{41}$ In addition, Zhu et al revealed that silencing UCA1 increased the expression of miR-18a and inhibited the expression of Yes-associated protein 1 (YAP1), a regulator of drug sensitivity, which could restore the chemo-sensitivity in trastuzumab-resistant SKBR-3 cells. ${ }^{42}$ Taken together, these findings demonstrated that UCA1 contributed to regulate the chemoresistance of $\mathrm{BC}$ cells.

\section{Esophageal cancer}

Jiao et al reported that UCA1 expression was elevated in esophageal cancer (EC) cells and tissues when compared with esophageal epithelial cell line NE1 and adjacent nontumor tissues. ${ }^{43}$ It was reported that lncRNAs can function as sponges of microRNA (miRNA) to regulate its activity. $^{44}$ The physical interaction between UCA1 and miR-204 was confirmed by RIP assay and luciferase assay. Furthermore, UCA1 acted as a sponge of miR-204 to positively regulate the expression of SOX4 that is a master mediator in tumorigenicity and cancer stemness to promote EC cell proliferation. The study also indicated amplified UCA1 was correlated with advanced TNM stage, higher clinical stage and poor prognosis, suggesting UCA1 might be a prognostic marker for EC patients.

\section{Lung cancer}

Recent studies have elucidated the role of UCA1 in lung cancer. According to a study by Nie et al, UCA1 was elevated in human non-small cell lung cancer (NSCLC) tissues. Kaplan-Meier analysis revealed that lung cancer patients with higher UCA1 expression level had poor clinical outcome. Functionally, silencing UCA1 inhibited NSCLC cell proliferation ability via sponging miR-193a$3 p$ to abolish its endogenous suppressive effect on ERBB4. ${ }^{45} \mathrm{Wu}$ et al also reported that UCA1 exerted oncogenes activity and modulated the proliferation and invasion of lung cancer cells. Subsequent mechanism studies demonstrated that UCA1 directly regulated miR-193a to increase the expression of HMGB1 that could function as a carcinogene in tumors. ${ }^{46}$ Thus, these studies manifested that UCA1 promoted tumorigenesis and its overexpression contributed to the progression of lung cancer.

\section{Prostate cancer}

Studies on UCA1 in prostate cancer (PCa) showed that it was significantly evaluated in PCa tissues and cell lines. Chemo- and radio-resistance remain obstacles for the failure of treatment in patients with PCa. Wang et al discovered that upregulation of UCA1 suppressed the activity of miR-204 and positively adjust sirt1 expression. The apoptosis induced by docetaxel was enhanced in docetaxel-resistant 22RV1/DR cells transfected with UCA1 siRNA, sirt1 siRNA and miR-204 mimics, namely UCA1 overexpression increased docetaxel chemoresistance via miR-204/sirt1 axis. ${ }^{47}$ Subsequently, Ghiam et al found UCA1 was significantly upregulated in prostate cancer radioresistant cells compared with parental cells. Further research suggested that knockdown UCA1 not only could reverse radioresistance, induce cell cycle arrest in $\mathrm{G} 2 / \mathrm{M}$ phase but also reduce the level of p-Akt, indicating $\mathrm{PI} 3 \mathrm{~K} / \mathrm{AKT}$ pathway was activated in the development of $\mathrm{PCa}$ radioresistance. ${ }^{48}$ Taken together, UCA1 also could influence the generation of resistance in prostate cancer.

\section{Pancreatic cancer}

An increasing number of studies showed that overexpression of UCA1 played important roles in biological processes of pancreatic cancer (PC). Zhang et al elucidated reduction of UCA1 decreased cell growth, migration, invasion ability in vitro and in vivo. Subsequently, rescue experiments manifested that miR-135a partially offset the effect of UCA1 on invasion and migration in PC cells. ${ }^{49}$ Additionally, a study by Zhou et al reported that UCA1 could bind miR-96 to modulate the expression of FOXO3 that may promote proliferation, metastases and reduce apoptosis of PC cells, indicating that the UCA1/miR-96/ FOXO3 axis participated in PC progression. ${ }^{50}$ Chen et al discovered that UCA1 inhibited the expression of P27 to regulate cell cycle and proliferation of PC cells. Patients with elevated UCA1 expression had shorter overall survival. $^{51}$ Therefore, these findings suggested that UCA1 participated in the development of PC, and it might be a significant independent predictor of poor survival.

\section{Ovarian cancer}

Studies on UCA1 in ovarian cancer (OC) revealed that UCA1 was highly expressed in ovarian tissues compared with the normal tissues, and elevated UCA1 in the patients 
respond to chemotherapy always had worse survival time. ${ }^{52}$ Similarly, a recent study indicated that UCA1 was upregulated in paclitaxel-resistant OC cells. Mechanistically, UCA1 acted as a sponge of miR-129 to promote the expression of $\mathrm{ABCB} 1$ which contributed to paclitaxel resistance. ${ }^{53}$ UCA1 was associated with chemoresistance and overall survival rate, suggesting that it might be a potential prognostic marker in ovarian cancer.

\section{Tongue squamous cell carcinoma}

A report by Fang et al demonstrated that UCA1 was highly expressed in tongue squamous cell carcinoma (TSCC) tissues, especially in tissues with lymph node metastasis. ${ }^{54}$ Consistent with the Fang et al study, Zhang et al further found that TGF- $\beta 1$ stimulated the expression of UCA1. Mechanistically, the researchers discovered that UCA1 competitively bound to miR-124 to decrease its inhibitory effect on downstream JAG1 and Notch1, which could promote EMT process and invasion of TSCC. $^{55}$ In general, these results demonstrated that UCA1 acted as an oncogene in TSCC progression.

\section{Melanoma}

When compared with normal controls, Tian et al and Wei et al identified that UCA1 expression was obviously elevated in melanoma tissues. Moreover, Tian et al suggested UCA1 might have a correlation with metastasis, and it is highly expressed in melanomas with lymph node metastasis than those at early stage. ${ }^{56}$ In addition, Wei et al also further confirmed that knockdown UCA1 inhibited cell proliferation and induced G0/G1 cell cycle arrest by directly interacting with miR-507 to affect FOXM1 expression. ${ }^{57}$ Therefore, UCA1 was involved in cell proliferation, metastasis and cell cycle arrest, it could be considered as a potential therapeutic target.

\section{Thyroid carcinoma}

$\mathrm{Lu}$ et al have studied the effects of UCA1 on thyroid cancer cells, the researchers showed that highly expressed UCA1 could promote proliferation and invasion, while inhibit apoptosis of thyroid cancer cells. ${ }^{58} \mathrm{~A}$ report by Liu et al demonstrated that UCA1 regulated the expression of IGFBP5 by acting as a ceRNA to sponge miR-204 in papillary thyroid carcinoma cells. ${ }^{59}$ In summary, these studies established a potential role of UCA1 in the development of thyroid carcinoma.

\section{Osteosarcoma}

The expression and mechanisms of UCA1 in the osteosarcoma have been studied by Zhu et al, the researchers indicated that knockdown UCA1 inhibited cell growth and migration by regulation of miR-301a and CXCR4. This study further confirmed Wnt and NF- $\mathrm{kB}$ signaling pathways were activated when upregulation CXCR $4 .{ }^{60} \mathrm{Li}$ et al reported that transcriptional factor HIF- $1 \alpha$ could induce UCA1 expression and promote cell growth of osteosarcoma. Moreover, the author then examined whether UCA1 mediated cell growth and cell cycle arrest through the PTEN/AKT signaling pathway. The results showed that UCA1 suppressed PTEN expression while increased p-AKT expression to regulate cell growth. ${ }^{61}$ Taken together, UCA1 upregulation is crucial in the pathogenesis of osteosarcoma.

\section{Glioma}

Recent studies have elucidated the role of UCA1 in glioma, showing this lncRNA was upregulated in glioma patient tissues and cell lines and associated with poor prognosis. Sun et al confirmed UCA1 promotes the development of glioma by decreasing the expression of tumor suppressor miR-122. ${ }^{62}$ Similarly, He et al studied the interaction between UCA1 and miRNAs, and found that UCA1 could directly sponge miR-182 to enhance inhibitor of apoptosis-stimulating protein of p53 (IASPP) expression, demonstrating that UCA1/miR-182/ IASPP axis participated in glioma progression. ${ }^{63}$ These findings demonstrated that upregulated UCA1 possessed prognostic value and contributed to the progression of glioma.

\section{Multiple myeloma}

There are few published studies concerning the expression of UCA1 in multiple myeloma (MM). In multiple myeloma tissues and cell lines, Zhang et al confirmed that UCA1 expression was markedly evaluated. ${ }^{64}$ High expression of UCA1 promoted cell proliferation, suppressed cell apoptosis and was associated with poor overall survival in patients with MM. Mechanistically, UCA1 could upregulate oncogene TGF- $\beta$ expression to promote carcinogenesis. Therefore, UCA1 might be an oncogenic lncRNA in $\mathrm{MM}$, suggesting its potential utilities as a prognostic marker. However, further studies on the role of UCA1 in $\mathrm{MM}$ development are warranted. 


\section{Oral squamous cell carcinoma}

A report by Yang et al found that UCA1 was obviously upregulated in oral squamous cell carcinoma (OSCC) cell lines compared with normal oral cell line. Furthermore, mechanistic analysis revealed that $\mathrm{Wnt} / \beta$-catenin signaling pathway was activated when UCA1 overexpression, with cell proliferation, migration and invasion promotion and cell apoptosis inhibition. ${ }^{65}$ Moreover, the connection between UCA1 and cisplatin (CDDP) chemoresistance of oral squamous cell carcinoma was explored by Fang et al. Highly expressed UCA1 existed in CDDP-resistant OSCC cells, it enhanced proliferation, decreased CDDP susceptibility and inhibited CDDP-induced apoptosis of OSCC cells. Molecular mechanisms study further elucidated that UCA1 upregulated SF1 expression by acting as a ceRNA to affect the expression of miR-184. ${ }^{66}$ Thus, these data revealed that UCA1 knockdown effectively suppressed cell proliferation, migration and invasion and enhanced CDDP susceptibility in OSCC.

\section{Cervical cancer}

As for cervical cancer, the present study discovered that UCA1 expression was dramatically increased in cervical cancer tissues. Yan et al discovered that UCA1 directly interacted with miR-206 to increase VEGF expression, which enhanced the migration and invasion capability of cervical cancer cells. ${ }^{67}$ In addition, Fan et al examined the relationship between UCA1 and radioresistance of cervical cancer cells, the researchers found that UCA1 was significantly elevated in radioresistant cell lines that exhibited increased glycolysis. Further studies found that UCA1 promoted HK2 expression to enhance glucose consumption and lactate production, and ultimately increased the radioresistance of cervical cancer cells. ${ }^{68}$ Therefore, UCA1 could act as a functional oncogene, and it was expected to regard as a novel prognosis target in cervical cancer.

In summary, these studies indicated that UCA1 was highly expressed in human cancers, and associated with cell proliferation, migration, invasion, apoptosis, chemoand radio-resistance, and EMT process. In the progress of bladder cancer, colorectal cancer, pancreatic cancer, hepatocellular carcinoma, thyroid carcinoma, etc., these findings suggested UCA1 functioned as a ceRNA to modulate miRNAs and target genes expression, thus promoting tumorigenesis. In addition, in the development of gastric cancer, breast cancer, prostate cancer, osteosarcoma, etc., UCA1 activated several critical signaling pathways, such as Wnt/ $\beta$ catenin, PI3K/AKT, NF- $\mathrm{KB}$ signaling pathway, to enhance biological processes, including ability of cell proliferation, migration, invasion, and chemo- and radio-resistance. Moreover, increased UCA1 usually had poor clinicopathological features and uncomfortable outcome, suggesting UCA1 was closely associated with prognosis, thus, it was expected to serve as a promising predictor for poor prognosis of cancer patients.

\section{Regulating mechanisms of UCAI Function as a ceRNA}

Mounting evidence has demonstrated competing endogenous RNA (ceRNA), an important underlying mechanism in various cancer development, can serve as sponges of miRNAs to modulate its target genes expression. ${ }^{26-28}$ Bian et al suggested that UCA1 could sponge endogenous miR-204-5p and inhibit its activity, enhancing the expression of CREB1, BCL2 and RAB22A (Figure 1A). ${ }^{30}$ Another study found that knockdown UCA1 repressed the proliferation, migration and metastasis capacity in esophageal cancer via binding miR-204 to promote the expression of SOX4 that contributes to tumorigenicity (Figure 1B). ${ }^{43}$ Similarly, Zhou et al reported that UCA1 directly interacted with miR-96 to regulate FOXO3 level, showing that UCA1/miR-96/FOXO3 axis participated in pancreatic cancer progression. ${ }^{50}$

\section{Involved in signaling pathways EMT signaling pathway}

EMT is an early event of tumor invasion and metastasis, the typical performance of which is downregulation of epithelial cell marker E-cadherin expression and upregulation of stromal cell markers such as N-cadherin, fibronectin and $\beta$-catenin. ${ }^{69,70}$ Xue et al revealed that highly expressed UCA1 in bladder cancer could decrease E-cadherin expression while increase vimentin expression, suggesting UCA1 promoted tumorigenesis through modulating EMT (Figure 1C). ${ }^{23}$ HMGB1, a member of the high mobility group box subfamily, is an inducer of EMT, Luo et al demonstrated overexpression of UCA1 could competitively bind to miR-143, and thus increase the expression level of HMGB1 and subsequently induce EMT signaling pathway activation (Figure 1D). ${ }^{24}$

\section{ERK signaling pathway}

ERK, which included ERK1 and ERK2, is one of the most common signaling pathways in the MAPK family that is involved in several cellular biological responses such as cell proliferation, differentiation, transformation and 


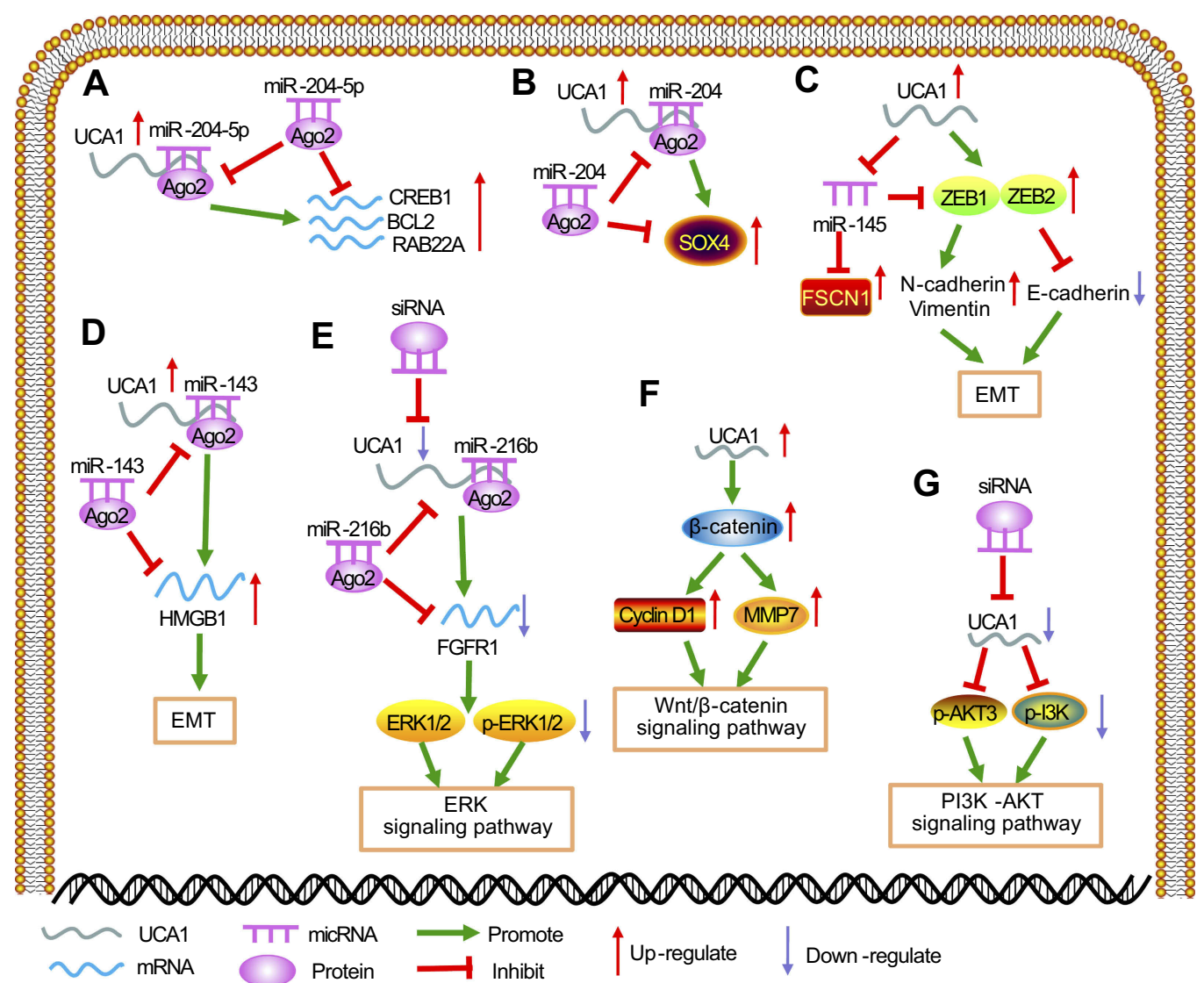

Figure I Regulating mechanisms of UCAI in multiple human cancers. (A) UCAI acted as a ceRNA to bind with miR-204-5p to increase the expression of CREBI, BCL2 and RAB22A. (B) UCAI functioned as a ceRNA to bind with miR-204 to upregulate SOX4 expression level. (C) Increased UCAI could upregulate N-cadherin and vimentin expression, while E-cadherin, an epithelial marker, was downregulated. (D) UCAI served as an endogenous sponge of miR-I43 to increase the expression of HMGBI then promote EMT process. (E) Silencing UCAI could repress the expression of FGFR I to inhibit the activation of ERK signaling pathway. (F) UCAI could regulate the expression of $\boldsymbol{\beta}$-catenin to activate $\mathrm{Wnt} / \boldsymbol{\beta}$-catenin signaling pathway. (G) The PI3K/AKT signaling pathway was activated when UCAI was highly expressed.

apoptosis. $^{71}$ A study reported that FGFR1 could act as an upstream factor of the MAPK signaling pathway to regulate its cascade. ${ }^{72}$ Wang et al showed that UCA1 could bind with miR-216b to promote FGFR1 expression, further research found that depletion of UCA1 inhibited the expression of ERK1 and ERK2, indicating high expressed UCA1 could activate ERK signaling pathway (Figure 1E). ${ }^{38}$

\section{Wnt/ $\beta$-catenin signaling pathway}

The $\beta$-catenin protein, widely present in eukaryotic cells, was abnormal activated in tumorigenesis. As shown in study by Liu et al, $\beta$-catenin was aggregated and transferred to the nucleus in chemoresistance breast cancer cells (MCF-7-R and T47D-R). In addition, it was found that $\beta$ catenin located in the cytoplasm when suppressed lncRNA UCA1, suggesting that UCA1 could contribute to the $\beta$ catenin cytonuclear translocation. ${ }^{40}$ At the same time, in the study of breast cancer, Xiao et al also reported that overexpressed UCA1 upregulated nuclear $\beta$-catenin expression level and increased transcription of cyclin D1 and MMP-7 that are downstream genes of $\beta$-catenin (Figure $1 \mathrm{~F}){ }^{73}$ These data demonstrated that the $\mathrm{Wnt} / \beta$ catenin signaling pathway was inhibited when silencing UCA1.

\section{PI3K/AKT pathway}

The PI3K/AKT signaling pathway is usually abnormal activated and regulates cell proliferation and progression in many human cancers. Experimental conducted by Li et al and Qin et al revealed that amplified UCA1 enhanced the expression of p-PI3K and p-AKT3, suggesting the PI3K/ AKT pathway was abnormal activated (Figure 1G). ${ }^{32,33}$ Similarly, Ghiam et al also found that silencing UCA1 reduced the expression level of p-Akt that could increase chemosensitivity, suggesting UCA1 could activate the PI3K/AKT signaling pathway to act oncogenic effect. ${ }^{48}$ These findings indicated that UCA1 may play a pivotal role in the development of various cancers. 


\section{Epigenetic and transcriptional regulation Histone methylation modulation}

An accumulation of studies have indicated that UCA1 regulates the transcription of genes by epigenetic modifications. A study found that UCA1 facilitated to hepatocellular tumorigenesis through physically binding to enhancer of EZH2 and suppressed transcription of P27 by histone methylation (H3K27me3) on its promoter, resulting in upregulation of CDK2 that is crucial in cell cycle progression (Figure 2A). ${ }^{74}$ Lee et al suggested that UCA1 was upregulated when SATB1 depletion, with H3K4 trimethylation (H3K4me3) levels increased and H3K27 trimethylation (H3K27me3) level decreased, these indicated that SATB1 repressed the expression of UCA1 and further suppressed growth and survival of breast cancer cells. ${ }^{75}$ Taken together, several studies demonstrated that UCA1 manipulated transcriptional regulation through histone methylation on the promoter of genes.

\section{Transcriptional factor and complex}

Wang et al showed that transcription factor SP1 directly bound to the core promoter of UCA1 to increase its expression, then UCA1 physically interacted with EZH2 to stimulate the translation of cyclin D1, promoting the transition of gastric cancer cells into G1/S and facilitating to cell proliferation (Figure 2B). ${ }^{76}$ The Hippo pathway is closely related to the TGF- $\beta$ - SMAD pathway. ${ }^{77}$ As a report by Mota et al, a tumor suppressor Merlin was downregulated in breast cancer tissues, resulting in inactivation of Hippo pathway and translocation of YAP/TAZ into the nucleus. The YAP/ TAZ in the nucleus not only could bind with the transcription factor TEAD but also interact with the SMAD complex in the TGF- $\beta$-SMAD pathway to activate downstream target gene UCA1 that involved in tumorigenesis, cell proliferation, migration and invasion (Figure 2C). ${ }^{78}$ Similarly, Zhang et al found UCA1 could interact with the mediators MOB1, Lats1 and YAP of the Hippo pathway to form a ribonucleoprotein complex, and increase nuclear localization and stabilization of YAP, promoting cell proliferation and inhibiting apoptosis in pancreatic cancer. ${ }^{79}$ These studies indicated transcription factors and complexes could regulate the expression of UCA1 by binding with their promoters.

\section{Conclusion and future perspectives}

In the present, a growing number of evidence has reported that IncRNAs, regulators of human gene expression, were abnormal expressed and influenced the development of cancers. LncRNA UCA1, a typical oncogene, was observed overexpressed in various cancers. Amplified UCA1 could affect many cellular functions, such as contributing to cell proliferation, migration, invasion, while restraining cell apoptosis in vitro and in vivo. The underlying regulatory mechanisms of UCA1 were explored and involved multiple steps, including competitively binding with miRNAs to regulate related genes expression, abnormal activation of several significant signaling pathways, and alteration of epigenetic and transcriptional regulation. These studies also revealed that UCA1 could be regarded as a prognosis
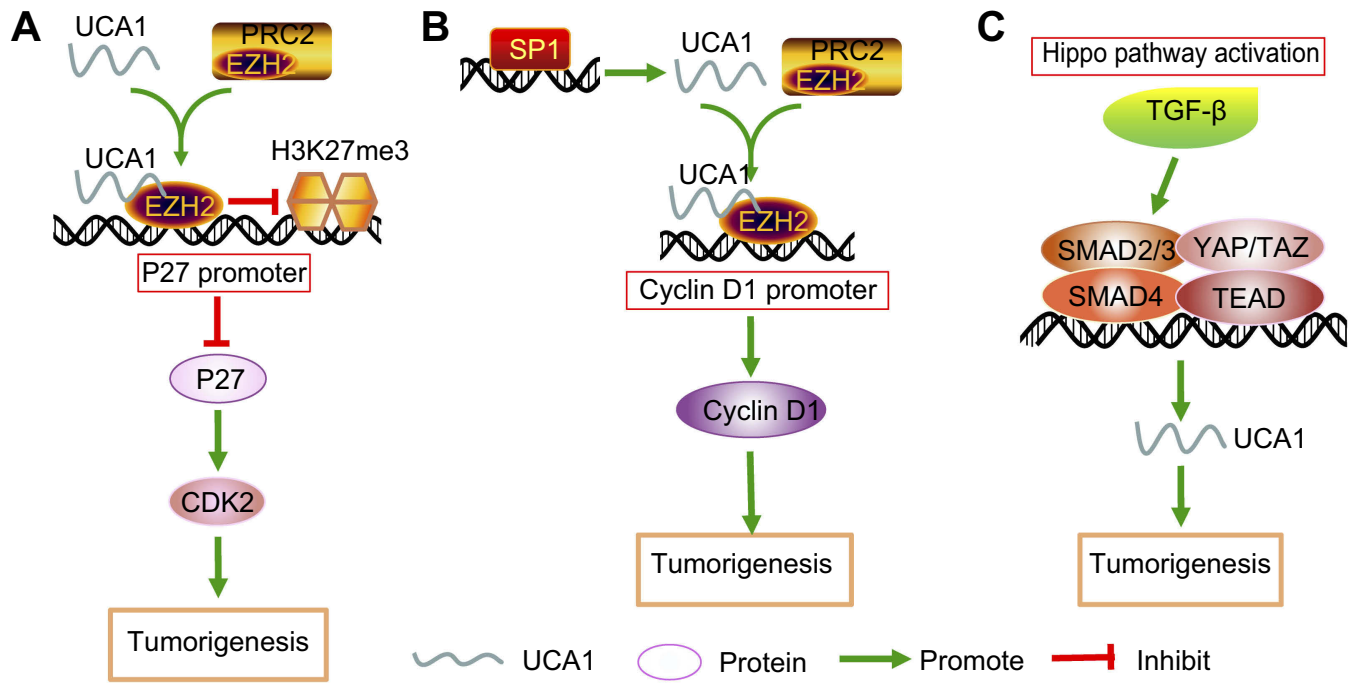

Figure 2 Epigenetic and transcriptional regulation mechanisms of UCAI in multiple human cancers. (A) UCAI physically bound to EZH2 and suppressed transcription of P27 by histone methylation (H3K27me3) on the promoter of p27KipI. (B) Transcription factor SPI could activate UCAI expression, and UCAI physically combined with EZH2 to enhance the expression of cyclin DI. (C) TEAD SMAD complexes promoted the level of UCAI. 
biomarker due to highly expression of it was related to poor clinicopathological features especially for shorter overall survival. However, further studies are needed to elucidate the prognostic value of UCA1 in human cancers.

Although lncRNA has received more and more attention, and further studies are carried out in various tumors, but compared with mRNA and miRNA, the detailed mechanisms about lncRNA in tumorigenesis and development have still not been fully explained. In other words, due to the diversity and complexity of IncRNA mechanisms, the current understanding of lncRNA function is still in primary stage, which requires more research on UCA1 to explore the regulatory mechanisms in carcinogenesis in the future.

In summary, UCA1 can be regarded as an oncogene to influence tumor progression and a promising predictor for poor prognosis for cancer patients, further works will be necessary for facilitating translation of UCA1 from basic research into clinical utility as early as possible.

\section{Acknowledgment}

This study was funded by Health Commission of Hubei Province Scientific Research Project (WJ2019M256).

\section{Disclosure}

The authors report no conflicts of interest in this work.

\section{References}

1. Torre LA, Freddie B, Siegel RL, et al. Global cancer statistics, 2012. CA Cancer J Clin. 2015;65(2):69-90. doi:10.3322/caac.21262

2. Koo MM, Hamilton W, Walter FM, et al. Symptom signatures and diagnostic timeliness in cancer patients: a review of current evidence. Neoplasia. 2018;20(2):165-174. doi:10.1016/j.neo.2017.11.005

3. Nie J, Zhao C, Deng LI, et al. Efficacy of traditional Chinese medicine in treating cancer. Biomed Rep. 2016;4(1):3-14. doi:10.3892/ br.2015.537

4. Nakashima L. Evolution of cancer treatment and evolving challenges. Healthc Manage Forum. 2018;31(1):26-28. doi:10.1177/ 0840470417722568

5. Hajjari M, Khoshnevisan A, Shin YK. Molecular function and regulation of long non-coding RNAs: paradigms with potential roles in cancer. Tumor Biol. 2014;35(11):10645-10663. doi:10.1007/s13277-014-2636-Z

6. Tang Y, Cheung BB, Atmadibrata B, et al. The regulatory role of long noncoding RNAs in cancer. Cancer Lett. 2017;391:12-19. doi:10.1016/j.canlet.2017.01.010

7. Guo H, Huang S, Li S, et al. Prognostic significance of the long noncoding RNAs in nasopharyngeal carcinoma: a systematic review and meta-analysis. Cancer Manag Res. 2018;10:1763-1779. doi:10.2147/CMAR.S164695

8. Iakes E, David J, Jose Manuel R, et al. Multiple evidence strands suggest that there may be as few as 19,000 human protein-coding genes. Hum Mol Genet. 2014;23(22):5866-5878. doi:10.1093/hmg/ddu309
9. Yamamura S, Imai-Sumida $\mathrm{M}$, Tanaka $\mathrm{Y}$, et al. Interaction and cross-talk between non-coding RNAs. Cell Mol Life Sci. 2018;75 (3):467-484. doi:10.1007/s00018-017-2626-6

10. Yang S, Sun Z, Zhou Q, et al. MicroRNAs, long noncoding RNAs, and circular RNAs: potential tumor biomarkers and targets for colorectal cancer. Cancer Manag Res. 2018;10:2249-2257. doi:10.2147/ CMAR.S166308

11. Quinn JJ, Chang HY. Unique features of long non-coding RNA biogenesis and function. Nat Rev Genet. 2015;17(1):47-62. doi:10.1038/nrg.2015.10

12. Schmitt A, Chang H. Long noncoding RNAs in cancer pathways. Cancer Cell. 2016;29(4):452-463. doi:10.1016/j.ccell.2016.03.010

13. Huang J, Zhou N, Watabe K, et al. Long non-coding RNA UCA1 promotes breast tumor growth by suppression of p27 (Kip1). Cell Death Dis. 2014;5(1):e1008. doi:10.1038/cddis.2013.541

14. Wang X, Fa XE. Knockdown of UCA1 inhibits viability and glycolysis by suppressing PKM2 expression through the mTOR pathway in non-small cell lung cancer cells. RSC $A d v$. 2018;8 (19):10610-10619. doi:10.1039/C8RA00860D

15. Xie XJ, Xu LI, Wang F. Cellular localization and tissue expression pattern of UCA1, a non-coding RNA. J South Med Univ. 2010;30 (1):57-60. doi:10.3724/SP.J.1008.2010.01244

16. Wang XS, Zhang Z, Wang HC, et al. Rapid identification of UCA1 as a very sensitive and specific unique marker for human bladder carcinoma. Clin Cancer Res. 2006;12(16):4851-4858. doi:10.1158/ 1078-0432.CCR-06-0134

17. Xue M, Chen W, Li X. Urothelial cancer associated 1: a long noncoding RNA with a crucial role in cancer. $J$ Cancer Res Clin Oncol. 2016;142(7):1-13. doi:10.1007/s00432-015-2042-y

18. Yazarlou F, Modarressi MH, Mowla SJ, et al. Urinary exosomal expression of long non-coding RNAs as diagnostic marker in bladder cancer. Cancer Manag Res. 2018;10:6357-6365. doi:10.2147/ CMAR.S186108

19. Li Z, Dou P, Liu T, He S. Application of long noncoding RNAs in osteosarcoma: biomarkers and therapeutic targets. Cell Physiol Biochem. 2017;42(4):1407-1419. doi:10.1159/000479205

20. Sun XD, Chen H, Wei Q, et al. Clinical significance of UCA1 to predict metastasis and poor prognosis of digestive system malignancies: a meta-analysis. Gastroenterol Res Pract. 2016;2016(1):1-11. doi: $10.1155 / 2016 / 3729830$

21. Song W, Gobe GC. Understanding molecular pathways and targets of Brachyury in epithelial-mesenchymal transition (EMT) in human cancers. Curr Cancer Drug Targets. 2016;16(7):586-593. doi:10.2174/1568009616666160328113338

22. Chen YC, Statt S, Wu R, et al. High mobility group box 1-induced epithelial mesenchymal transition in human airway epithelial cells. Sci Rep. 2016;6:18815. doi:10.1038/srep18815

23. Xue M, Pang H, Li X, et al. Long non-coding RNA urothelial cancer-associated 1 promotes bladder cancer cell migration and invasion by way of the hsa-miR-145-ZEB1/2-FSCN1 pathway. Cancer Sci. 2016;107(1):18-27. doi:10.1111/cas.12844

24. Luo J, Chen J, Li H, et al. LncRNA UCA1 promotes the invasion and EMT of bladder cancer cells by regulating the miR-143/HMGB1 pathway. Oncol Lett. 2017;14(5):5556-5562. doi:10.3892/ ol.2017.6886

25. Gou L, Liu M, Xia J, et al. BMP9 promotes the proliferation and migration of bladder cancer cells through up-regulating lncRNA UCA1. Int J Mol Sci. 2018;19(4):1116. doi:10.3390/ ijms 19041116

26. Tay Y, Rinn J, Pier Paolo P. The multilayered complexity of ceRNA crosstalk and competition. Nature. 2014;505(7483):344-352. doi:10.1038/nature12986

27. Cesana M, Cacchiarelli D, Legnini I, et al. A long noncoding RNA controls muscle differentiation by functioning as a competing endogenous RNA. Cell. 2011;147(2):358-369. doi:10.1016/j. cell.2011.09.028 
28. Zhao G, Fu Y, Su Z, et al. How long non-coding RNAs and microRNAs mediate the endogenous RNA network of head and neck squamous cell carcinoma: a comprehensive analysis. Cell Physiol Biochem. 2018;50(1):332-341. doi:10.1159/000494009

29. Zheng C, Li X, Qian B, et al. The lncRNA myocardial infarction associated transcript-centric competing endogenous RNA network in non-small-cell lung cancer. Cancer Manag Res. 2018;10:1155-1162. doi:10.2147/CMAR.S163395

30. Bian Z, Jin L, Zhang J, et al. LncRNA-UCA1 enhances cell proliferation and 5-fluorouracil resistance in colorectal cancer by inhibiting miR-204-5p. Sci Rep. 2016;6:23892. doi:10.1038/srep23892

31. Ni B, Yu X, Guo X, et al. Increased urothelial cancer associated 1 is associated with tumor proliferation and metastasis and predicts poor prognosis in colorectal cancer. Int J Oncol. 2015;47(4):1329-1338. doi:10.3892/ijo.2015.3109

32. Li C, Liang G, Yang S, et al. Dysregulated lncRNA-UCA1 contributes to the progression of gastric cancer through regulation of the PI3K-Akt-mTOR signaling pathway. Oncotarget. 2017;8 (55):93476-93491. doi:10.18632/oncotarget.19281

33. Qin L, Jia Z, Xie D, Liu Z. Knockdown of long noncoding RNA urothelial carcinoma-associated 1 inhibits cell viability, migration, and invasion by regulating microRNA-182 in gastric carcinoma. $J$ Cell Biochem. 2018;119(12):10075-10086. doi:10.1002/jcb.27344

34. Gong P, Qiao F, Wu H, et al. LncRNA UCA1 promotes tumor metastasis by inducing miR-203/ZEB2 axis in gastric cancer. Cell Death Dis. 2018;9(12):1158. doi:1038/s41419-018-1170-0

35. Gu L, Lu LS, Zhou DL, et al. UCA1 promotes cell proliferation and invasion of gastric cancer by targeting CREB1 sponging to miR-5903p. Cancer Med. 2018;7(4):1253-1263. doi:10.1002/cam4.1310

36. Li J, Gao J, Kan A, et al. SNHG and UCA1 as prognostic molecular biomarkers in hepatocellular carcinoma: recent research and meta-analysis. Minerva Med. 2017;108(6):568-574. doi:10.23736/ S0026-4806.17.05094-7

37. Qin LT, Tang RX, Lin P, et al. Biological function of UCA1 in hepatocellular carcinoma and its clinical significance: investigation with in vitro and meta-analysis. Pathol Res Pract. 2018;214 (9):1260-1272. doi:10.1016/j.prp.2018.03.025

38. Wang F, Ying HQ, He BS, et al. Upregulated 1ncRNA-UCA1 contributes to progression of hepatocellular carcinoma through inhibition of miR-216b and activation of FGFR1/ERK signaling pathway. Oncotarget. 2015;6(10):7899-7917. doi:10.18632/oncotarget.3219

39. Xiao JN, Yan TH, Yu RM, et al. Long non-coding RNA UCA1 regulates the expression of Snail2 by miR-203 to promote hepatocellular carcinoma progression. J Cancer Res Clin Oncol. 2017;143 (6):981-990. doi:10.1007/s00432-017-2370-1

40. Liu H, Wang G, Yang L, et al. Knockdown of long non-coding RNA UCA1 increases the tamoxifen sensitivity of breast cancer cells through inhibition of $\mathrm{Wnt} / \beta$-catenin pathway. PLOS One. 2016;11 (12):e0168406. doi:10.1371/journal.pone.0168406

41. Wu C, Luo J. Long non-coding RNA (lncRNA) Urothelial Carcinoma-Associated 1 (UCA1) enhances tamoxifen resistance in breast cancer cells via inhibiting mTOR signaling pathway. Med Sci Monit. 2016;22:3860-3867. doi:10.12659/msm.900689

42. Zhu HY, Bai WD, Ye XM, et al. Long non-coding RNA UCA1 desensitizes breast cancer cells to trastuzumab by impeding miR-18a repression of Yes-associated protein 1. Biochem Biophys Res Commun. 2018;496(4):1308-1313. doi:10.1016/j. bbrc.2018.02.006

43. Jiao C, Song Z, Chen J, et al. IncRNA-UCA1 enhances cell proliferation through functioning as a ceRNA of Sox4 in esophageal cancer. Oncol Rep. 2016;36(5):2960-2966. doi:10.3892/or.2016.5121

44. Du Z, Sun T, Hacisuleyman E, et al. Integrative analyses reveal a long noncoding RNA-mediated sponge regulatory network in prostate cancer. Nat Commun. 2016;7:10982. doi:10.1038/ncomms10982
45. Nie W, Ge HJ, Yang XQ, et al. LncRNA- UCA1 exerts oncogenic functions in non-small cell lung cancer by targeting miR-193a-3p. Cancer Lett. 2016;371(1):99-106. doi:10.1016/j.canlet.2015.11.024

46. Wu H, Zhou C. Long non-coding RNA UCA1 promotes lung cancer cell proliferation and migration via microRNA-193a/HMGB1 axis. Biochem Biophys Res Commun. 2018;496(2):738-745. doi:10.1016/j. bbrc.2018.01.097

47. Wang X, Yang B, Ma B. The UCA1/miR-204/Sirt1 axis modulates docetaxel sensitivity of prostate cancer cells. Cancer Chemother Pharmacol. 2016;78(5):1025-1031. doi:10.1007/s00280-016-3158-8

48. Fotouhi GA, Taeb S, Huang X, et al. Long non-coding RNA urothelial carcinoma associated 1 (UCA1) mediates radiation response in prostate cancer. Oncotarget. 2016;8(3):4668-4689. doi:10.18632/ oncotarget. 13576

49. Zhang X, Gao F, Zhou L, et al. UCA1 regulates the growth and metastasis of pancreatic cancer by sponging MiR-135a. Oncol Res. 2017;25(9):1529-1541. doi:10.3727/096504017X14888987683152

50. Zhou Y, Chen Y, Ding W, et al. LncRNA UCA1 impacts cell proliferation, invasion, and migration of pancreatic cancer through regulating miR-96/FOXO3. IUBMB Life. 2018;70(4):276-290. doi:10.1002/iub.1699

51. Chen P, Wan D, Zheng D, et al. Long non-coding RNA UCA1 promotes the tumorigenesis in pancreatic cancer. Biomed Pharmacother. 2016;83:1220-1226. doi:10.1016/j.biopha.2016.08.041

52. Zhang L, Cao X, Zhang L, Zhang X, Sheng H, Tao K. UCA1 overexpression predicts clinical outcome of patients with ovarian cancer receiving adjuvant chemotherapy. Cancer Chemother Pharmacol. 2016;77(3):629-634. doi:10.1007/s00280-016-2963-4

53. Wang J, Ye C, Liu J, Hu Y. UCA1 confers paclitaxel resistance to ovarian cancer through miR-129/ABCB1 axis. Biochem Biophys Res Commun. 2018;501(4):1034-1040. doi:10.1016/j.bbrc.2018.05.104

54. Fang Z, Wu L, Wang L, Yang Y, Meng Y, Yang H. Increased expression of the long non-coding RNA UCA1 in tongue squamous cell carcinomas: a possible correlation with cancer metastasis. Oral Surg Oral Med Oral Pathol Oral Radiol. 2014;117(1):89-95. doi:10.1016/j.oooo.2013.09.007

55. Zhang TH, Liang LZ, Liu XL, et al. LncRNA UCA1/miR-124 axis modulates TGF $\beta 1$-induced epithelial-mesenchymal transition and invasion of tongue cancer cells through JAG1/Notch signaling. $J$ Cell Biochem. 2019;120(6):10495-10504. doi:10.1002/jcb.28334

56. Tian Y, Zhang X, Hao Y, Fang Z, He Y. Potential roles of abnormally expressed long noncoding RNA UCA1 and Malat-1 in metastasis of melanoma. Melanoma Res. 2014;24(4):335-341. doi:10.1097/ CMR.0000000000000080

57. Wei Y, Sun Q, Zhao L, et al. LncRNA UCA1-miR-507-FOXM1 axis is involved in cell proliferation, invasion and $\mathrm{G} 0 / \mathrm{G} 1$ cell cycle arrest in melanoma. Med Oncol. 2016;33(8):88. doi:10.1007/s12032-016-0804-2

58. Lu HW, Liu XD. UCA1 promotes papillary thyroid carcinoma development by stimulating cell proliferation via Wnt pathway. Eur Rev Med Pharmacol Sci. 2018;22(17):5576-5582. doi:10.26355/ eurrev 20180915821

59. Liu H, Li R, Guan L, Jiang T. Knockdown of lncRNA UCA1 inhibits proliferation and invasion of papillary thyroid carcinoma through regulating miR-204/IGFBP5 axis. Onco Targets Ther. 2018;11:7197-7204. doi:10.2147/OTT.S175467

60. Zhu G, Liu X, Su Y, Kong F, Hong X, Lin Z. Knockdown of Urothelial Carcinoma-Associated 1 suppressed cell growth and migration through regulating miR-301a and CXCR4 in osteosarcoma MHCC97 cells. Oncol Res. 2018;27(1):55-64. doi:10.3727/ $096504018 X 15201143705855$

61. Li T, Xiao Y, Huang T. HIF-1 $\alpha$-induced upregulation of lncRNA UCA1 promotes cell growth in osteosarcoma by inactivating the PTEN/AKT signaling pathway. Oncol Rep. 2018;39(3):1072-1080. doi:10.3892/or.2018.6182 
62. Sun Y, Jin JG, Mi WY, Zhang SR, Meng Q, Zhang ST. Long noncoding RNA UCA1 targets miR-122 to promote proliferation, migration, and invasion of glioma cells. Oncol Res. 2018;26 (1):103-110. doi:10.3727/096504017X14934860122864

63. He Z, Wang Y, Huang G, Wang Q, Zhao D, Chen L. The IncRNA UCA1 interacts with miR-182 to modulate glioma proliferation and migration by targeting iASPP. Arch Biochem Biophys. 2017;623-624:1-8. doi:10.1016/j.abb.2017.01.013

64. Zhang ZS, Wang J, Zhu BQ, Ge L. Long noncoding RNA UCA1 promotes multiple myeloma cell growth by targeting TGF- $\beta$. Eur Rev Med Pharmacol Sci. 2018;22(5):1374-1379. doi:10.26355/ eurrev_201803_14481

65. Yang YT, Wang YF, Lai JY, et al. Long non-coding RNA UCA1 contributes to the progression of oral squamous cell carcinoma by regulating the WNT/ $\beta$-catenin signaling pathway. Cancer Sci. 2016;107(11):1581-1589. doi:10.1111/cas.13058

66. Fang Z, Zhao J, Xie W, Sun Q, Wang H, Qiao B. LncRNA UCA1 promotes proliferation and cisplatin resistance of oral squamous cell carcinoma by sunppressing miR-184 expression. Cancer Med. 2017;6 (12):2897-2908. doi:10.1002/cam4.1253

67. Yan Q, Tian Y, Hao F. Downregulation of lncRNA UCA1 inhibits proliferation and invasion of cervical cancer cells through miR-206 expression. Oncol Res. 2018. doi:10.3727/096504018X15 185714083446

68. Fan L, Huang C, Li J, Gao T, Lin Z, Yao T. Long noncoding RNA urothelial cancer associated 1 regulates radioresistance via the hexokinase 2/glycolytic pathway in cervical cancer. Int $J$ Mol Med. 2018;42(4):2247-2259. doi:10.3892/ijmm.2018.3778

69. Pang A, Carbini M, Moreira AL, et al. Carcinosarcomas and related cancers: tumors caught in the act of epithelial-mesenchymal transition. $J$ Clin Oncol. 2017;36(2):210-216. doi:10.1200/ JCO.2017.74.9523

70. Feng G, Kazuko H, Sen-Itiroh H. Specific glycosphingolipids mediate epithelial-to-mesenchymal transition of human and mouse epithelial cell lines. Proc Natl Acad Sci U S A. 2009;106 (18):7461-7466. doi:10.1073/pnas.0902368106
71. Sun Y, Liu WZ, Liu T, et al. Signaling pathway of MAPK/ERK in cell proliferation, differentiation, migration, senescence and apoptosis. J Recept Signal Transduct Res. 2015;35(6):600-604. doi:10.3109/10799893.2015.1030412

72. Dienstmann R, Rodon J, Prat A, et al. Genomic aberrations in the FGFR pathway: opportunities for targeted therapies in solid tumors. Ann Oncol. 2014;25(3):552-563. doi:10.1093/annonc/mdu203

73. Xiao $\mathrm{C}, \mathrm{Wu} \mathrm{CH}, \mathrm{Hu} \mathrm{HZ}$. LncRNA UCA1 promotes epithelialmesenchymal transition (EMT) of breast cancer cells via enhancing Wnt/ $\beta$-catenin signaling pathway. Eur Rev Med Pharmacol Sci. 2016;20(13):2819-2824.

74. Hu JJ, Song W, Zhang SD, et al. HBx-upregulated lncRNA UCA1 promotes cell growth and tumorigenesis by recruiting EZH2 and repressing p27Kip1/CDK2 signaling. Sci Rep. 2016;6:23521. doi:10.1038/srep23521

75. Lee JJ, Kim M, Kim HP. Epigenetic regulation of long noncoding RNA UCA1 by SATB1 in breast cancer. BMB Rep. 2016;49(10):578-583. doi:10.5483/bmbrep.2016.49.10.156

76. Wang ZQ, Cai Q, Hu L, et al. Long noncoding RNA UCA1 induced by SP1 promotes cell proliferation via recruiting EZH2 and activating AKT pathway in gastric cancer. Cell Death Dis. 2017;8(6):e2839. doi: $10.1038 /$ cddis. 2017.518

77. Ge M, Liu H, Zhang Y, et al. The anti-hepatic fibrosis effects of dihydrotanshinone I are mediated by disrupting the yes-associated protein and transcriptional enhancer factor D2 complex and stimulating autophagy. $\mathrm{Br} \quad J$ Pharmacol. 2017;174(10):1147-1160. doi:10.1111/bph.13766

78. Hiemer SE, Szymaniak AD, Varelas X. The transcriptional regulators TAZ and YAP direct transforming growth factor $\beta$-induced tumorigenic phenotypes in breast cancer cells. J Biol Chem. 2014;289 (19):13461-13474. doi:10.1074/jbc.M113.529115

79. Zhang M, Zhao Y, Zhang Y, et al. LncRNA UCA1 promotes migration and invasion in pancreatic cancer cells via the Hippo pathway. Biochim Biophys Acta Mol Basis Dis. 2018;1864(5 Pt A):1770-1782. doi:10.1016/j.bbadis.2018.03.005

\section{Publish your work in this journal}

Cancer Management and Research is an international, peer-reviewed open access journal focusing on cancer research and the optimal use of preventative and integrated treatment interventions to achieve improved outcomes, enhanced survival and quality of life for the cancer patient.
The manuscript management system is completely online and includes a very quick and fair peer-review system, which is all easy to use. Visit http://www.dovepress.com/testimonials.php to read real quotes from published authors. 